\title{
Coordinated Standoff Tracking Using Path Shaping for Multiple UAVs
}

\author{
Hyondong Oh, Dario Turchi, Seungkeun Kim, \\ Antonios Tsourdos, Lorenzo Pollini, and Brian White
}

\begin{abstract}
This paper presents a coordinated standoff target tracking strategy using path shaping for multiple UAVs. In performing a tracking mission of a ground target of interest, UAVs are to approach a target and to keep a standoff distance from it with a prescribed inter-vehicle angular separation around the target in order to track it while acquiring accurate target information. Since fixed-wing UAVs without hovering capability fly efficiently at a nominal airspeed, it is desirable that they can keep angular separation between vehicles while holding a constant velocity in a mission duration point of view. This paper introduces a new path shaping technique using two constant curvature segments satisfying the turn radius constraint and having more flexibility and fewer discontinuous points on a curvature command compared to the Dubins path at the expense of the path length. Moreover, a simultaneous arrival concept is introduced as a coordinated tracking strategy for multiple UAVs, which initialises UAVs on a standoff orbit with a desired angular separation. In order to address arrival time delay or failure of the UAV, a two-orbit approach is proposed in which UAVs first arrive at the outer orbit and subsequently shrink to the desired inner orbit at different time, while adjusting the angular separation between constant-speed UAVs. The feasibility and benefits of the proposed approach are evaluated via numerical simulations for a ground target using two cooperative UAVs.
\end{abstract}

Index Terms-UAVs, Standoff Tracking, Path Shaping, Simultaneous Arrival, Angular Separation

\section{INTRODUCTION}

Monitoring and subsequent tracking of a moving ground target of interest is one of the important capabilities of UAV(Unmanned Aerial Vehicle)s required to increase situational awareness and to take proactive measures against unknown intents of the threat [1], [2], [3], [4]. In performing such missions, UAVs are to keep a certain distance from the target known as a standoff distance while maintaining a prescribed inter-vehicle angular separation in order to track it without being noticed from the target and simultaneously to maximize target information acquisition. An optimal configuration of UAVs is to place vehicles at either equal angular spacing around a target [5] or a certain angular position in the orbit depending on the number of vehicles and sensor characteristics [6], [7]. For this tracking mission, Frew et

Hyondong Oh is with Department of Computing, University of Surrey, Guildford, GU2 7XH, United Kingdom, e-mail: h.oh@surrey.ac.uk. Dario Turchi, Antonios Tsourdos and Brian White are with the Department of Engineering Physics, Cranfield University, Cranfield, MK43 0AL, United Kingdom, e-mail: \{d.turchi, a.tsourdos, b.a.white\}@cranfield.ac.uk. Seungkeun Kim is with the Department of Aerospace Engineering, Chungnam National University, Daejeon, 305-764, Korea, email: skim78@cnu.ac.kr. Lorenzo Pollini is with the Dipartimento di Sistemi Elettrici e Automazione, Facolta di Ingegnerià, Università di Pisa, 56126 Pisa, Italy, email: lpollini@dsea.unipi.it. al. [8] proposed the application of Lyapunov vector fields for standoff coordination of multiple UAVs. They invented a decoupled control structure in which speed and turn rate are separately controlled for standoff distance and phase angle keeping, respectively. Summers et al. [9] extended this phasekeeping idea to multiple UAVs using information architectures in vehicle formations. Moreover, they addressed a variable airspeed controller to maintain the circular orbit despite unknown wind and unknown constant velocity target motion using adaptive estimation. Similarly, Kingston et al. [10] used a vector field approach, however, they introduced a sliding mode control and orbit radius change without velocity change for phase-keeping of multiple UAVs. Sepulchre et al. [11] and Paley et al. [12] introduced the notion of the splay state which represents uniform rotation of evenly spaced vehicles on a circle. They applied the collective control of multiagent system to stabilize symmetric circular formation around the target using unit speed vehicles. Klein and Morgansen [13] proposed a steering control law making the velocity of the collective centroid match a reference velocity allowing tracking of a moving target. Wise and Rysdyk [14] extensively surveyed and compared the different methodologies for standoff tracking: Helmsman behavior, Lyapunov vector field, controlled collective motion, and model predictive control. Recently, Kim et al. [15] and Oh et al. [16] proposed a model predictive guidance for coordinated standoff tracking considering realistic car trajectory data in urban traffic.

While the existing approaches aforementioned have been focusing on the guidance law design changing turn rate and speed, this paper proposes a novel path shaping strategy taking kinematic constraints of multiple UAVs into account for a coordinated standoff target tracking. Since fixed-wing UAVs fly at a nominal airspeed for a longer duration with better fuel efficiency than a rotary-wing UAV with hovering capability, it is desirable that they keep angular separation between vehicles while holding a constant velocity. In order to produce flyable paths to fit to the fixed-wing UAV dynamics, the proposed path shaping algorithm takes into account the most critical constraint of fixed-wing UAVs: curvature or radius of turning maneuver, which is dependent on the operating range of speed and bank angle. Moreover, it is advantageous for the shaped paths to have a shorter length as well as lower curvature differences at discontinuous points as possible in order to minimize flight time or energy consumption [17]. For this, a path shaping algorithm uses two constant curvature segments in this paper. As a measure to coordinated target tracking for multiple UAVs, a simultaneous arrival concept 
is introduced with the proposed path shaping, as part of an outer layer approach that initialises UAVs on a standoff orbit with a desired angular separation. In order to address arrival time delay or failure of the UAV, a two-orbit approach is proposed in which UAVs first arrive at the outer orbit and subsequently shrink to the desired inner orbit at different time, while adjusting the angular separation between constant-speed UAVs.

As a main contribution, the proposed standoff tracking strategy is more realistic because it is based on the constant-speed maneuvers of fixed-wing UAVs, whereas most of the previous research, except for Sepulchre et al. [11] and Paley et al. [12], considers speed variations especially for phase separation between the UAVs. Another contribution is that using path shaping for standoff tracking can consider constraints of UAV kinematics (i.e. curvature or turn radius) inherently, generating feasible paths for UAVs at all times; whereas the existing literature often need to limit a turn rate or curvature command from the guidance loop to the saturation values. Also, this paper newly derives the path shaping with two constant curvature segments. The proposed algorithm using two arc segments can be regarded as having more flexibility and fewer discontinuity points on a curvature command compared to the widely-used Dubins path [18] composed of three consecutive combinations of circular arc and line segments. Fewer discontinuities in a guidance command are desirable for fixed-wing UAVs since they cannot exactly follow a discontinuous curvature (or bank angle) command (which occurs twice for the Dubins path and once for the proposed method) and, in reality, can track an approximated continuous command. Moreover, Monte Carlo simulation results in Section IV justify that the proposed method provides even less curvature differences at those discontinuous points compared with the Dubins path at the expense of path length. In other words, the proposed approach provides a longer path length but with fewer discontinuity points as well as lower curvature differences than those of the Dubins path. This is the first case to apply a path shaping strategy to coordinated standoff tracking by multiple UAVs.

Note that, this study considers the path shaping approach using kinematic constraints of the UAV, providing an openloop path. For real implementation, feedback control to follow the generated path is required to cope with gust and disturbances. However, the proposed approach still has an advantage over the other guidance approaches which requires sharing the information continuously via communication between UAVs for simultaneous arrival to the standoff orbit with the desired angular separation; in the proposed two-orbit approach, UAVs need to follow the generated path and communicate each other twice when each UAV reaches the outer orbit. The proposed techniques can be flexibly extended to produce the paths with longer lengths for the purpose of obstacle avoidance or to synchronize the arrival time of UAVs to the standoff circle around the target. To verify the feasibility and benefits of the proposed coordinated standoff tracking strategy, numerical simulations are performed for both stationary and moving ground targets using two cooperative UAVs.

The remainder of this paper is organized as follows. Section II defines the path shaping problem for the UAV, and proposed a new algorithm using constant curvature segments. The coordinated standoff target tracking strategy using the path shaping is then described in Section III. Lastly, the feasibility and benefits of the proposed algorithm is evaluated via numerical simulations in Section IV. Conclusions and future work are given in Section V.

\section{Path Shaping by Constant Curvature Segments}

This section defines the path shaping problem for the UAV, and proposed a new path shaping algorithm using constant curvature segments. In this paper, the path shaping represents connecting any two points of interest by a curve satisfying certain constraints, which aims to create an entire path for the standoff target tracking in a wide sense. The path shaping for the UAV should be able to produce a feasible path whose end points are characterized by position $(x, y)$ and orientation $\sigma$, together called a configuration $P(x, y, \sigma)$. A feasible path is both flyable, meeting the kinematic constraints and safe to fly without collision to each other or any other obstacles. Given initial and final configurations $P_{s}\left(x_{s}, y_{s}, \sigma_{s}\right)$ and $P_{f}\left(x_{f}, y_{f}, \sigma_{f}\right)$, respectively, maximum curvature $\kappa_{\max }$ (or minimum turning radius $r_{m}$ ) and the other constraints $\amalg$ such as obstacles, the path planner generates a path $r(t)$ with parameter $t$ as:

$P_{s}\left(x_{s}, y_{s}, \sigma_{s}\right) \stackrel{r(t)}{\longrightarrow} P_{f}\left(x_{f}, y_{f}, \sigma_{f}\right), \quad \kappa(t) \leq \kappa_{\max }$, and $\coprod$.

In the literature, different approaches have been used to generate the feasible path satisfying constraints of the UAV. Chandler et al. [19] used Voronoi diagram to produce routes for the UAVs by minimising radar detection, and the routes were then refined to make them flyable. Rapidly-Exploring Trees (RRTs) is also used for collision free paths, with a path smoothing algorithm based on cubic spiral curves to satisfy the minimum turning radius constraints in [20]. Optimisation techniques are also used such as probabilistic methods [21], MILP [22], and genetic algorithm [23], [24] to produce paths by optimising certain cost function. In these approaches, the final outcome is typically a route plan which satisfies defined constraints; if the route is refined by adding constraints to consider kinematics of the UAV, the resulted path is a series of lines and arcs, which is a subset of Dubins paths [18]. Dubins showed that the shortest path between two vectors in a plane and meets minimum bounds on turning radius is a composite path formed by the segments of line and circular arcs, term as the Dubins paths. A mathematical proof is provided by Dubins and further dealt with by others ([25], [26], [27], [28]). It is commonly accepted as a reasonably accurate kinematic model for aircraft motion planning problem ([29], [30]). Shanmugavel et al. [31] used the Dubins path set of curves as well as Pythagorean curves connecting the start and goal points for UAVs, based on differential geometry. Even though the path generation is straightforward, this approach uses iteration to satisfy the constraints. They also proposed the use of clothoid arcs which have ramp curvature profile providing a smooth curvature transition between the arcs and the line segment, in order to address the path discontinuities [32]. 
The proposed path shaping technique in this study uses constant curvature segments. It connects two configurations by using two arc segments of the constant curvature along with the consideration of a bound on the curvature. A single arc segment between two positions only has one degree of freedom: an arc curvature. This is not enough to be able to match the pose constraint at both positions since at least two degrees of freedom are necessary. Extra degrees of freedom are thus required to ensure $\mathcal{C}^{1}$ contact at initial and final positions, which represents that the first derivative is continuous at both points. One solution to increase the degrees of freedom is to introduce an intermediate vertex such that the line segment is replaced by two arc segments of different curvature as shown in Fig. 1 [33]. Note that this solution is not unique nor necessarily time optimal. The initial and final position are identified as $\mathbf{v}_{s}$ and $\mathbf{v}_{f}$ with the intermediate vertex given by $\mathbf{v}_{i}$. Hence, two arcs of different curvature will connect two configurations via intermediate vertex with $\mathcal{C}^{1}$ contact. Two orientations for the intermediate arcs are possible as shown in Fig. 1. Alternatively, the paths can be divided into four cases

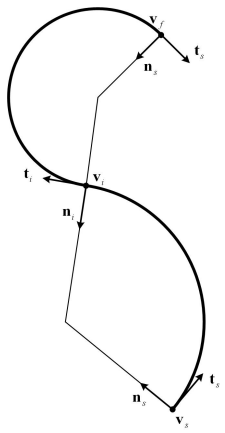

(a) Orientation 1

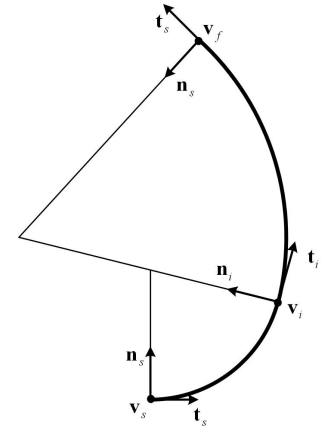

(b) Orientation 2
Fig. 1. Arc segments with $\mathcal{C}^{1}$ contact and intermediate vertex

based on the orientation of initial and final configurations as:

1) First case: initial and final orientations are towards $\mathbf{p}_{t}$,

2) Second case: initial and final orientations are opposite to $\mathbf{p}_{t}$,

3) Third case: the initial orientation is towards $\mathbf{p}_{t}$ while the final is opposite to it,

4) Fourth case: the initial orientation is opposite to $\mathbf{p}_{t}$ while the final is towards it,

where $\mathbf{p}_{t}$ represents a tangent intersection point of two orientations as shown in Fig. 2. Since the conditions for some of the cases are either identical or similar, only the first and the third case will be explained in detail from the following.

\section{A. First and second case}

The first case is defined as the one with initial and final orientations towards their tangent intersection point $\mathbf{p}_{t}$ as shown in Fig. 3. Using the angles inside the quadrilateral formed by points $\mathbf{p}_{t}, \mathbf{v}_{s}, \mathbf{v}_{f}$ and $\mathbf{v}_{i}$ gives angle $\theta$ as:

$$
\theta=2 \pi-\phi_{s}-\phi_{f}-\psi
$$

where $\phi_{s}$ and $\phi_{f}$ represent the angles between the tangents and the chord $c$ connecting initial and final positions to

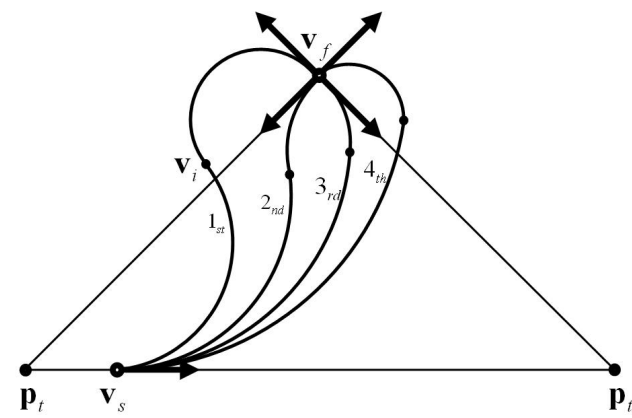

Fig. 2. Four possible cases of the paths using two arc segments

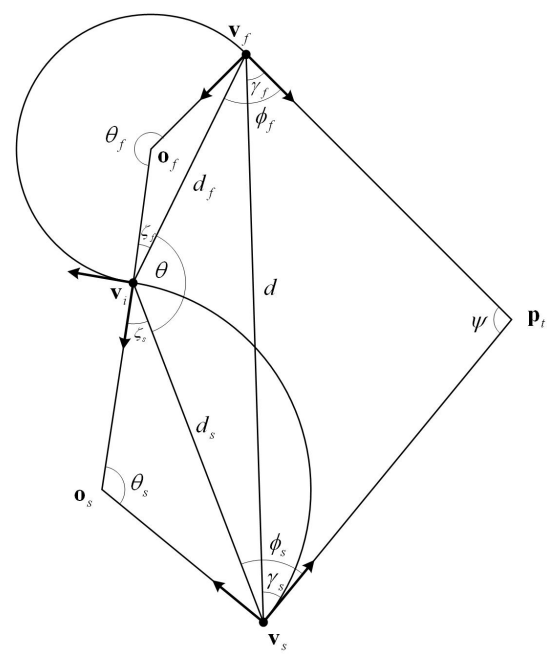

Fig. 3. Geometry for the first case

the intermediate vertex, respectively. Considering the triangle $\left(\mathbf{v}_{s}, \mathbf{v}_{i}, \mathbf{o}_{s}\right)$ and $\left(\mathbf{v}_{f}, \mathbf{v}_{i}, \mathbf{o}_{f}\right)$, angle $\theta$ can also be expressed as:

$$
\theta=\pi-\zeta_{s}-\zeta_{f}
$$

where $\zeta_{s}=\pi / 2-\phi_{s}$ and $\zeta_{f}=\pi / 2-\phi_{f}$. Combing Eq. (1) and Eq. (2) gives:

$$
\phi_{f}=\pi-\psi / 2-\phi_{s} .
$$

Both $\phi_{s} \geq 0$ and $\phi_{f} \geq 0$ are required to maintain consistent arc direction by orienting the directed tangent vector at the intermediate vertex, which gives a boundary for $\phi_{s}$ as:

$$
0 \leq \phi_{s} \leq \pi-\psi / 2
$$

Another limitation on the angle comes from the first case requirement itself, as given:

$$
-\pi<\theta_{s}-\theta_{f} \leq 0
$$

where angular values are subtracted since the direction of the turn maneuver applied in this case is opposite to each other. As shown in Fig. 4, given $\theta_{s}$, the dashed line where $\theta_{s}-\theta_{f}<0$ represents the first case with initial and final orientations towards their tangent intersection, while the solid line represents the limit condition for this case, where $\theta_{s}-\theta_{f}=0, \phi_{s}=\gamma_{s}$, 


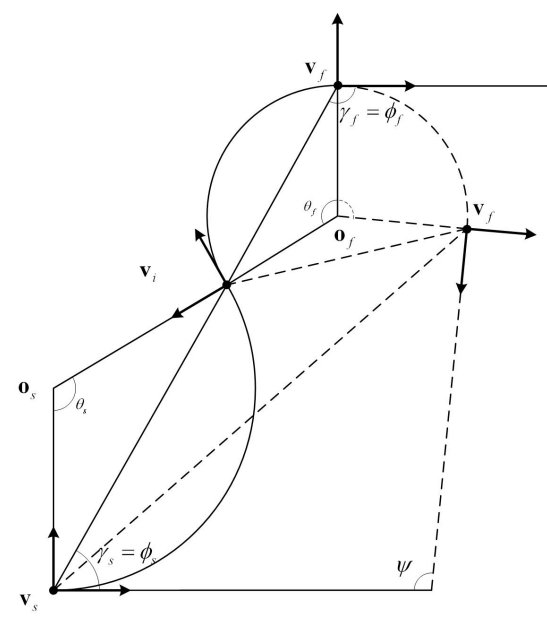

Fig. 4. Feasible and limit conditions for the first case

and $\phi_{f}=\gamma_{f}$. This relation gives the following inequality:

$$
\begin{aligned}
& \phi_{s} \geq \gamma_{s} \\
& \phi_{f} \geq \gamma_{f} .
\end{aligned}
$$

where $\gamma_{s}$ and $\gamma_{f}$ represent angles between tangents and the chord segment. Using Eq. (3) and Eq. (6), the final boundary of $\phi_{s}$ is obtained:

$$
\gamma_{s} \leq \phi_{s} \leq \pi-\psi / 2-\gamma_{f}
$$

To obtain other parameters for the generation of the path, the arc chord length $d_{s}$ and $d_{f}$ are first obtained using triangle $\left(\mathbf{v}_{s}, \mathbf{v}_{i}, \mathbf{v}_{f}\right)$ and sine rule as:

$$
d_{s}=d \frac{\sin \left(\phi_{f}-\gamma_{f}\right)}{\sin (\theta)} ; \quad d_{f}=d \frac{\sin \left(\phi_{s}-\gamma_{s}\right)}{\sin (\theta)} .
$$

where $d$ represents the vertex chord length between the vertices $\mathbf{v}_{s}$ and $\mathbf{v}_{f}$. Besides, the curvature of each segment $\kappa_{s}$ and $\kappa_{f}$ can be determined as:

$$
\kappa_{s}=2 \sin \left(\phi_{s}\right) / d_{s} ; \quad \kappa_{f}=2 \sin \left(\phi_{f}\right) / d_{f} .
$$

The path shaping technique explained above produces the path that consists of two arcs with different curvature values $\kappa_{s}$ and $\kappa_{f}$. However, in order to produce flyable paths for the UAV, physical constraints of the UAV flight, the limited curvature or the minimum turning radius, should be taken into account. In the following, these constraints are dealt with by changing the boundary on $\phi_{s}$, that is the only degree of freedom available, satisfying the bound on the curvature $\kappa_{\max }$ or turn radius $r_{m}$.

First of all, let us check the characteristic of the curvature or turning radius for initial arc depending on the value of $\phi_{s}$ using Eq. (3), Eq. (9) and Eq. (10) as:

$$
r_{s}=\frac{1}{\kappa_{s}}=d \frac{\sin \left(\phi_{f}-\gamma_{f}\right)}{2 \sin \theta \sin \phi_{s}}=d \frac{\sin \left(\alpha_{s_{1}}-\phi_{s}\right)}{2 \sin \theta \sin \phi_{s}}
$$

where $\alpha_{s_{1}}=\psi / 2+\gamma_{s}$. The characteristic of the turning radius for initial arc is obtained as the following lemma.

Lemma II.1. (Characteristic of the turning radius for the initial arc of 1st case): For $0<\psi<\pi, d>0$ and $0 \leq \phi_{s}<\pi$, initial turning radius $r_{s}$ is monotonically decreasing varying $\phi_{s}$ from its minimum to maximum bound in the first case.

Proof: Considering the boundary of $\phi_{s}$ from Eq. (8), $r_{s}$ for the minimum and the maximum bound of $\phi_{s}$ can be obtained as:

$$
\begin{aligned}
r_{s, \phi_{s_{\text {sup }}}} & =d \frac{\sin \left(\pi-\psi / 2-\phi_{s_{\text {sup }}}-\gamma_{f}\right)}{2 \sin \theta \sin \phi_{s_{\text {sup }}}}=0 \\
r_{s, \phi_{s_{\text {inf }}}} & =d \frac{\sin \left(\pi-\psi / 2-\phi_{s_{\text {inf }}}-\gamma_{f}\right)}{2 \sin \theta \sin \phi_{s_{i n f}}} \\
& =d \frac{\sin \psi / 2}{2 \sin \theta \sin \gamma_{s}}=d \frac{1}{2 \sin \gamma_{s}}
\end{aligned}
$$

where $\phi_{s_{\text {sup }}}=\pi-\psi / 2-\gamma_{f}, \phi_{s_{\text {inf }}}=\gamma_{s}$, and $\sin \theta=\sin \psi / 2$ since

$$
\sin \theta=\sin \left(2 \pi-\phi_{s}-\phi_{f}-\psi\right)=\sin \psi / 2 .
$$

Moreover, differentiating Eq. (11) with respect to $\phi_{s}$ gives:

$\frac{\partial r_{s}}{\partial \phi_{s}}=\frac{d}{2 \sin \theta} \frac{-\cos \left(\alpha_{s_{1}}-\phi_{s}\right) \sin \phi_{s}-\cos \phi_{s} \sin \left(\alpha_{s_{1}}-\phi_{s}\right)}{\sin ^{2} \phi_{s}}$.

It can be shown that Eq. (14) is always negative for $0<$ $\psi<\pi, d>0$ and $0 \leq \phi_{s}<\pi$ using simple trigonometry. Negative values for derivative, together with $r_{s}$ values for the minimum and the maximum bound of $\phi_{s}$ result in monotonically decreasing $r_{s}$ with increasing $\phi_{s}$.

Having analyzed the characteristic of the initial turning radius, a new limit on $\phi_{s}$ satisfying the bound on the turning radius $r_{m}$ can be obtained by imposing the following condition:

$$
r_{s}=d\left\{\sin \left(\phi_{f}-\gamma_{f}\right)\right\} /\left\{2 \sin \theta \sin \phi_{s}\right\} \geq r_{m} .
$$

Using Eq. (3) and trigonometry identities with Eq. (15) gives:

$$
\begin{array}{r}
\left\{\sin \left(\psi / 2+\phi_{s}+\gamma_{f}\right)\right\} /\left\{\sin \theta \sin \phi_{s}\right\} \geq 2 r_{m} / d \\
\cos \left(\psi / 2+\gamma_{f}\right)+\cot \phi_{s} \sin \left(\psi / 2+\gamma_{f}\right) \geq\left\{2 r_{m} \sin \psi / 2\right\} / d \\
\cot \phi_{s} \geq \frac{2 r_{m} \sin \psi / 2}{d \sin \left(\psi / 2+\gamma_{f}\right)}-\cot \left(\psi / 2+\gamma_{f}\right) .
\end{array}
$$

The feasible upper bound on $\phi_{s}$ to meet the turning radius constraint can be obtained as:

$$
\phi_{s} \leq \tan ^{-1}\left[1 /\left\{\beta_{s_{1}}-\cot \left(\psi / 2+\gamma_{f}\right)\right\}\right]
$$

where

$$
\beta_{s_{1}}=\left\{2 r_{m} \sin \psi / 2\right\} /\left\{d \sin \left(\psi / 2+\gamma_{f}\right)\right\} .
$$

The second part of the path characterized by the curvature $\kappa_{f}$ can be analyzed in the same manner as above: the turning radius of final arc $r_{f}$ tends to be a finite value for $\phi_{s_{s u p}}$, while it tends to be zero for $\phi_{s_{i n f}}$ as opposed to the previous initial arc case. By expressing $r_{f}$ as a function of $\phi_{f}$ gives:

$$
r_{f}=d \frac{\sin \left(\phi_{s}-\gamma_{s}\right)}{2 \sin \theta \sin \phi_{f}}=d \frac{\sin \left(\alpha_{f_{1}}-\phi_{f}\right)}{2 \sin \theta \sin \phi_{f}} .
$$

where $\alpha_{f_{1}}=\psi / 2+\gamma_{f}$. Then, the characteristic of the turning radius for final arc is obtained as the following lemma. 


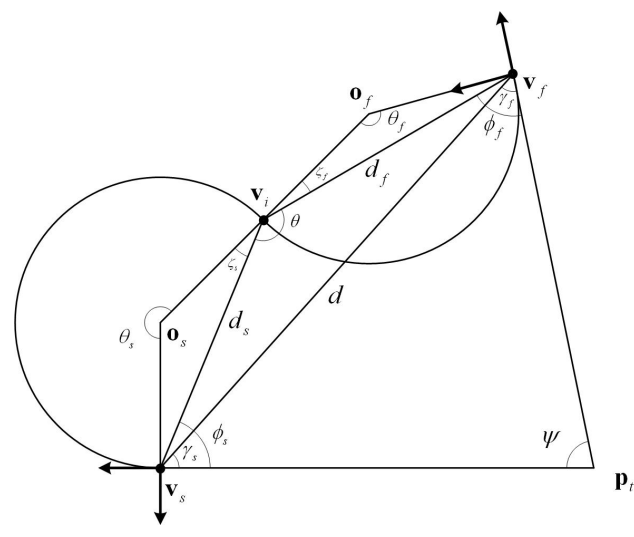

Fig. 5. Geometry for the second case

Lemma II.2. (Characteristic of the turning radius for the final arc of 1st case): For $0<\psi<\pi, d>0,0 \leq \phi_{s}<\pi$ and $0 \leq \phi_{f}<\pi$, final turning radius $r_{f}$ is monotonically decreasing with increasing $\phi_{f}$, but monotonically increasing with increasing $\phi_{s}$ in the first case.

Proof: It can be shown that $r_{f}$ is monotonically decreasing varying $\phi_{f}$ from its minimum to maximum bound using the same steps of the initial arc case. However, recalling that $\phi_{f}=\pi-\psi / 2-\phi_{s}$, it is clear that $\phi_{s}$ increases as $\phi_{f}$ decreases, resulting in monotonically increasing $r_{f}$ with increasing $\phi_{s}$.

To ensure the feasibility of the final arc solution, the following condition is imposed:

$$
r_{f}=d\left\{\sin \left(\phi_{s}-\gamma_{s}\right)\right\} /\left\{2 \sin \theta \sin \phi_{f}\right\} \geq r_{m} .
$$

The feasible upper bound on $\phi_{f}$ to meet the turning radius constraint can be obtained as:

$$
\phi_{f} \leq \tan ^{-1}\left[1 /\left\{\beta_{f_{1}}-\cot \left(\psi / 2+\gamma_{s}\right)\right\}\right]
$$

where

$$
\beta_{f_{1}}=\left\{2 r_{m} \sin \psi / 2\right\} /\left\{d \sin \left(\psi / 2+\gamma_{s}\right)\right\} .
$$

The feasible lower bound on $\phi_{s}$ can be obtained as:

$$
\begin{gathered}
\phi_{f}=\pi-\psi / 2-\phi_{s} \leq \tan ^{-1}\left[1 /\left\{\beta_{f_{1}}-\cot \left(\psi / 2+\gamma_{s}\right)\right\}\right] \\
\phi_{s} \geq-\tan ^{-1}\left[1 /\left\{\beta_{f_{1}}-\cot \left(\psi / 2+\gamma_{s}\right)\right\}\right]-\psi / 2+\pi .
\end{gathered}
$$

By choosing appropriate $\phi_{s}$ using the new boundary from Eq. (17) and Eq. (21) and following $\phi_{f}$ from Eq. (3), the flyable path for the UAV is generated.

The second case deals with the situation where both initial and final orientations are opposite to their tangent intersection point. An example of such a configuration is given in Fig. 5. A feasible solution requires:

$$
0<\theta_{s}-\theta_{f} \leq \pi .
$$

In this case, the geometrical configuration of triangle $\left(\mathbf{v}_{s}, \mathbf{v}_{i}, \mathbf{v}_{f}\right)$ and $\left(\mathbf{v}_{s}, \mathbf{p}_{t}, \mathbf{v}_{f}\right)$ does not change compared to the first case since initial and final positions are the same but with opposite orientations, and the only difference is rotation angles $\theta_{s}$ and $\theta_{f}$. By considering the fact that these angles do

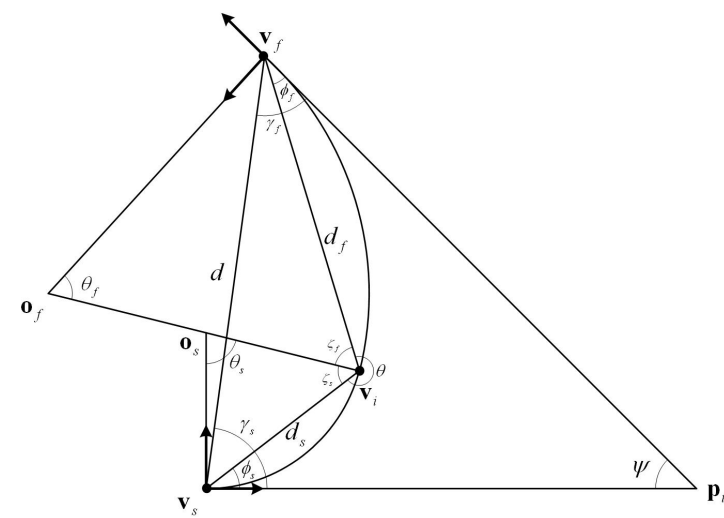

Fig. 6. Geometry for the third case

not affect the process of computing the parameters for the arc curvature, it can be concluded that the path constraints for the physical feasibility are obtained in a similar manner as for the first case.

To meet $\mathcal{C}^{1}$ contact at both positions and turning constraints, $\phi_{s}$ is selected appropriately within its boundary depending on the cost function such as the path length or any other metric as shown in Algorithm 1, which represents how to implement the proposed path shaping through pseudo-code.

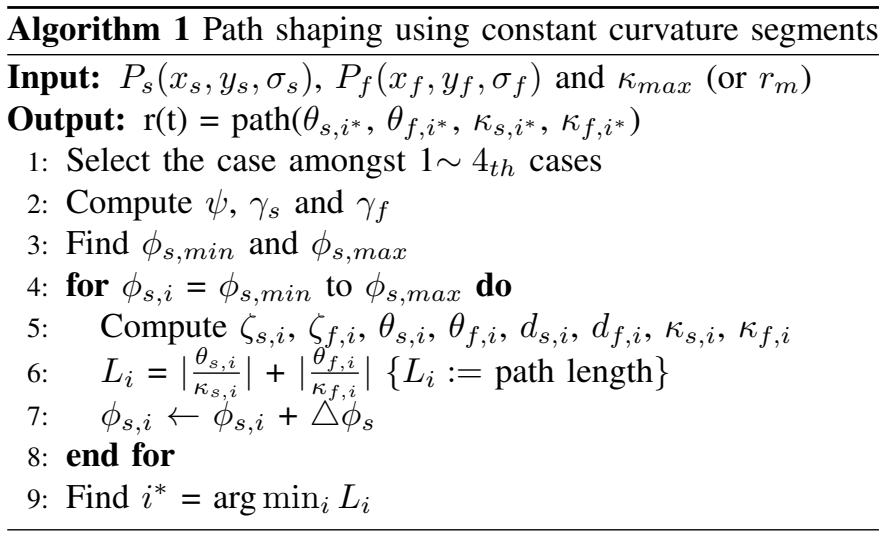

\section{B. Third and fourth case}

The third case deals with the situation where the initial orientation is towards tangent intersection point while the final is opposite to that. From the geometry as shown in Fig. 6, two different expressions for the angle $\theta$ can be obtained as:

$$
\begin{aligned}
\theta & =2 \pi-\phi_{s}-\phi_{f}-\psi \\
\theta & =2 \pi-\zeta_{s}-\zeta_{f}
\end{aligned}
$$

where $\zeta_{s}=\pi / 2-\phi_{s}$ and $\zeta_{f}=\pi / 2-\phi_{f}$. Combing above equations gives:

$$
\phi_{f}=\pi / 2-\psi / 2-\phi_{s} .
$$

Since the direction of two arcs is the same, the summation of the turning angle should satisfy:

$$
0<\theta_{s}+\theta_{f} \leq \pi
$$


leading to different boundary conditions from the one obtained in the first case as:

$$
\begin{aligned}
& 0 \leq \phi_{s} \leq \gamma_{s} \\
& 0 \leq \phi_{f} \leq \gamma_{f}
\end{aligned}
$$

Using Eq. (25) and Eq. (27), the final boundary of $\phi_{s}$ is given as:

$$
\begin{aligned}
& \phi_{s} \geq \max \left[0, \alpha_{s_{3}}\right] \\
& \phi_{s} \leq \min \left[\gamma_{s}, \pi / 2-\psi / 2\right]
\end{aligned}
$$

where $\alpha_{s_{3}}=\pi / 2-\psi / 2-\gamma_{f}$. Valid boundaries can be selected depending on required initial and final configurations. Considering the case where $\alpha_{s_{3}}=\pi / 2-\psi / 2-\gamma_{f} \leq 0$, the following relation holds:

$$
\begin{aligned}
\gamma_{s} & =\pi-\psi-\gamma_{f} \\
\gamma_{s} \leq \pi-\psi-\pi / 2+\psi / 2 & =\pi / 2-\psi / 2
\end{aligned}
$$

Similarly, the boundary for the case where $\alpha_{s_{3}}>0$ can be obtained, and combing with Eq. (29), the final boundary of $\phi_{s}$ is obtained more specifically as:

$$
\begin{array}{ll}
0 \leq \phi_{s} \leq \gamma_{s}, & \text { if } \alpha_{s_{3}} \leq 0 \\
\pi / 2-\psi / 2-\gamma_{f}<\phi_{s}<\pi / 2-\psi / 2, & \text { otherwise. }
\end{array}
$$

The arc chord length $d_{s}$ and $d_{f}$ are obtained using triangle $\left(\mathbf{v}_{s}, \mathbf{v}_{i}, \mathbf{v}_{f}\right)$ and sine rule, resulting in the same equation as Eq. (9):

$$
\begin{aligned}
& d_{s}=d \frac{\sin \left(\gamma_{f}-\phi_{f}\right)}{\sin (2 \pi-\theta)}=d \frac{\sin \left(\phi_{f}-\gamma_{f}\right)}{\sin (\theta)} \\
& d_{f}=d \frac{\sin \left(\gamma_{s}-\phi_{s}\right)}{\sin (2 \pi-\theta)}=d \frac{\sin \left(\phi_{s}-\gamma_{s}\right)}{\sin (\theta)} .
\end{aligned}
$$

Other parameters like the curvature can be obtained using the same way as in the first case. Moreover, to address the physical constraints, the turning radius is first expressed as a function of $\phi_{s}$ as done for the first case:

$$
r_{s}=d \frac{\sin \left(\phi_{f}-\gamma_{f}\right)}{2 \sin \theta \sin \phi_{s}}=d \frac{\sin \left(\phi_{s}-\alpha_{s_{3}}\right)}{2 \cos \psi / 2 \sin \phi_{s}} .
$$

The characteristic of the turning radius of the initial arc for the third case is obtained as the following lemma.

Lemma II.3. (Characteristic of the turning radius for the initial arc of 3rd case): For $0<\psi<\pi, d>0$ and $0 \leq \phi_{s}<\pi$, initial turning radius $r_{s}$ is monotonically decreasing with increasing $\phi_{s}$ for $\alpha_{s_{3}}<0$, and increasing for $\alpha_{s_{3}}>0$ in the third case.

Proof: Differentiating Eq. (34) with respect to $\phi_{s}$ gives:

$$
\frac{\partial r_{s}}{\partial \phi_{s}}=\frac{d}{2 \cos \theta} \frac{\cos \left(\phi_{s}-\alpha_{s_{3}}\right) \sin \phi_{s}-\cos \phi_{s} \sin \left(\phi_{s}-\alpha_{s_{3}}\right)}{\sin ^{2} \phi_{s}} .
$$

In case that $\alpha_{s_{3}}<0$, since $\phi_{s} \in\left(0, \gamma_{s}\right)$ from Eq. (32), the following holds:

$$
\begin{aligned}
\left(\phi_{s}-\alpha_{s_{3}}\right)_{\text {inf }} & =-\alpha_{s_{3}}>0 \\
\left(\phi_{s}-\alpha_{s_{3}}\right)_{\text {sup }} & =\gamma_{s}-\pi / 2+\psi / 2+\gamma_{f}<\pi / 2 .
\end{aligned}
$$

When $\alpha_{s_{3}}>0$, since $\phi_{s} \in\left(\pi / 2-\psi / 2-\gamma_{f}, \pi / 2-\psi / 2\right)$ from Eq. (32), the boundary of $\phi_{s}-\alpha_{s_{3}}$ is obtained as:

$$
\begin{aligned}
& \left(\phi_{s}-\alpha_{s_{3}}\right)_{i n f}=0 \\
& \left(\phi_{s}-\alpha_{s_{3}}\right)_{\text {sup }}=\gamma_{f}
\end{aligned}
$$

which implies $\left(\phi_{s}-\alpha_{s_{3}}\right) \in(0, \pi)$. For $d>0$ and $\psi<\pi$, the sign of Eq. (35) depends on its second numerator as:

$$
\begin{aligned}
& \cos \left(\phi_{s}-\alpha_{s_{3}}\right) \sin \phi_{s}-\cos \phi_{s} \sin \left(\phi_{s}-\alpha_{s_{3}}\right) \\
= & \sin \phi_{s} \sin \left(\phi_{s}-\alpha_{s_{3}}\right)\left(\cot \left(\phi_{s}-\alpha_{s_{3}}\right)-\cot \phi_{s}\right) .(
\end{aligned}
$$

For $\alpha_{s_{3}}<0$, by recalling that $\phi_{s} \in(0, \pi),\left(\phi_{s}-\alpha_{s_{3}}\right) \in$ $(0, \pi / 2)$, and the cotangent function is monotonically decreasing in such an interval, it can be concluded that the sign of Eq. (36) is negative since $\phi_{s}<\phi_{s}-\alpha_{s_{3}}$. Similarly, for $\alpha_{s_{3}}>0$, since $\phi_{s}>\phi_{s}-\alpha_{s_{3}}$, the sign of Eq. (36) is positive, resulting in:

$$
\begin{aligned}
& \partial r_{s} / \partial \phi_{s}<0, \text { if } \alpha_{s_{3}}<0 \\
& \partial r_{s} / \partial \phi_{s}>0, \text { if } \alpha_{s_{3}}>0
\end{aligned}
$$

Once monotonically increasing or decreasing behavior is determined, the boundary of $\phi_{s}$ for the feasible path can be obtained by imposing the following condition:

$$
\begin{gathered}
r_{s}=d\left\{\sin \left(\phi_{s}-\alpha_{s_{3}}\right)\right\} /\left\{2 \cos \psi / 2 \sin \phi_{s}\right\} \geq r_{m} \\
\frac{\sin \phi_{s} \cos \alpha_{s_{3}}-\cos \phi_{s} \sin \alpha_{s_{3}}}{\sin \phi_{s}} \geq \frac{2 r_{m} \cos \psi / 2}{d} \\
-\cot \phi_{s} \sin \alpha_{s_{3}} \geq\left\{2 r_{m} \cos \psi / 2\right\} / d-\cos \alpha_{s_{3}} .
\end{gathered}
$$

Depending on the sign of $\alpha_{s_{3}}$, the feasible bounds on $\phi_{s}$ are obtained as:

$$
\begin{array}{ll}
\phi_{s} \leq \tan ^{-1}\left[1 /\left\{\beta_{s_{3}}+\cot \alpha_{s_{3}}\right\}\right], & \text { if } \alpha_{s_{3}} \leq 0 \\
\phi_{s}>\tan ^{-1}\left[1 /\left\{\beta_{s_{3}}+\cot \alpha_{s_{3}}\right\}\right], & \text { otherwise }
\end{array}
$$

where

$$
\beta_{s_{3}}=-\left\{2 r_{m} \cos \psi / 2\right\} /\left\{d \sin \alpha_{s_{3}}\right\} .
$$

Similar steps can be done for the final arc. Using $\alpha_{s_{3}}=\pi / 2-$ $\psi / 2-\gamma_{f}=-\pi / 2+\psi / 2+\gamma_{s}$ and Eq. (25) gives:

$$
\begin{aligned}
& \partial r_{f} / \partial \phi_{s}<0, \text { if } \alpha_{s_{3}}<0 \text { or } \alpha_{f_{3}}>0 \\
& \partial r_{f} / \partial \phi_{s}>0, \text { if } \alpha_{s_{3}}>0 \text { or } \alpha_{f_{3}}<0
\end{aligned}
$$

where $\alpha_{f_{3}}=\pi / 2-\psi / 2-\gamma_{s}$, and another upper boundary for $\phi_{s}$ as:

$$
\begin{array}{ll}
\phi_{s} \leq \pi / 2-\psi / 2-\tan ^{-1}\left[\frac{1}{\beta_{f_{3}}+\cot \alpha_{f_{3}}}\right], & \text { if } \alpha_{s_{3}} \leq 0 \\
\phi_{s}>\pi / 2-\psi / 2-\tan ^{-1}\left[\frac{1}{\beta_{f_{3}}+\cot \alpha f_{3}}\right], & \text { otherwise }
\end{array}
$$

where

$$
\beta_{f_{3}}=-\left\{2 r_{m} \cos \psi / 2\right\} /\left\{d \cos \left(\pi / 2+\gamma_{s}\right)\right\} .
$$

Note that for $\alpha_{s_{3}} \leq 0$, since both $r_{s}$ and $r_{f}$ decrease with increasing $\phi_{s}$ from Eq. (41) and Eq. (45), only upper bound on $\phi_{s}$ can be obtained using turning radius constraints for the initial and final arc; for $\alpha_{s_{3}} \geq 0$, only lower bound can be computed. Therefore, by choosing the appropriate $\phi_{s}$ using 


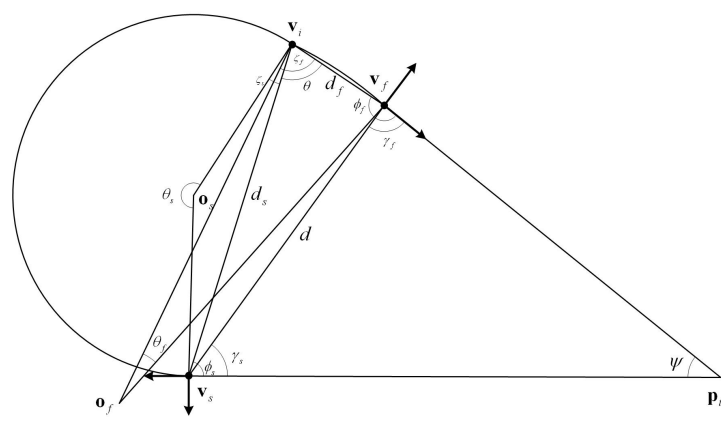

Fig. 7. Geometry for the fourth case

the boundary conditions from Eq. (32), Eq. (44), Eq. (47) and corresponding $\phi_{f}$ from Eq. (25), the flyable path for the UAV is generated.

The fourth case deals with the situation where the initial orientation is opposite to tangent intersection point while the final is towards that. An example of such a configuration is given in Fig. 7. A feasible solution requires:

$$
\pi<\theta_{s}+\theta_{f} \leq 2 \pi
$$

which gives the boundary of $\phi_{s}$ using a similar way as explained for the third case as:

$$
\begin{array}{ll}
\gamma_{s} \leq \phi_{s} \leq \pi, & \text { if } \alpha_{s_{4}} \leq 0 \\
\pi / 2-\psi / 2<\phi_{s}<\frac{3}{2} \pi-\psi / 2-\gamma_{f}, & \text { otherwise }
\end{array}
$$

where $\alpha_{s_{4}}=\gamma_{s}-\pi / 2+\psi / 2$. Similarly, the feasible bounds on $\phi_{s}$ from initial arc condition can be obtained as:

$$
\begin{array}{ll}
\phi_{s} \leq \tan ^{-1}\left[\frac{1}{\beta_{s_{4}}+\tan \left(\psi / 2+\gamma_{f}\right)}\right], & \text { if } \alpha_{s_{4}} \leq 0 \\
\phi_{s}>\tan ^{-1}\left[\frac{1}{\beta_{s_{4}}+\tan \left(\psi / 2+\gamma_{f}\right)}\right], & \text { otherwise }
\end{array}
$$

where

$$
\beta_{s_{4}}=-\left\{2 r_{m} \cos \psi / 2\right\} /\left\{d \cos \left(\psi / 2+\gamma_{f}\right)\right\} .
$$

The other feasible bound on $\phi_{s}$ from the final arc condition is obtained as:

$$
\begin{aligned}
& \phi_{s} \leq \frac{3}{2} \pi-\psi / 2-\tan ^{-1}\left[\frac{1}{\beta_{s_{4}}+\tan \left(\psi / 2+\gamma_{s}\right)}\right], \text { if } \alpha_{s_{4}} \leq 0 \\
& \phi_{s}>\frac{3}{2} \pi-\psi / 2-\tan ^{-1}\left[\frac{1}{\beta_{s_{4}}+\tan \left(\psi / 2+\gamma_{s}\right)}\right] \text {, }
\end{aligned}
$$

where

$$
\beta_{f_{4}}=-\left\{2 r_{m} \cos \psi / 2\right\} /\left\{d \cos \left(\psi / 2+\gamma_{s}\right)\right\} .
$$

By choosing the appropriate $\phi_{s}$ using the boundary conditions from Eq. (49), Eq. (50) and Eq. (51), the flyable path for the UAV is generated.

Given the boundary conditions and turning constraints, reachable space can be investigated by expressing the chord length $d$ from the triangle $\left(\mathbf{v}_{s}, \mathbf{v}_{i}, \mathbf{v}_{f}\right)$ in Fig. 7 as:

$$
\begin{aligned}
d^{2}= & d_{s}{ }^{2}+d_{f}{ }^{2}-2 d_{s} d_{f} \cos \theta \\
= & 4 r_{s}{ }^{2}\left(1-\cos \theta_{s}\right)+4 r_{f}{ }^{2}\left(1-\cos \theta_{f}\right) \\
& -8 r_{s} r_{f}\left(1-\cos \theta_{s}\right)\left(1-\cos \theta_{f}\right) \cos \theta .
\end{aligned}
$$

Then, the minimum value of $d$ for a feasible path with the minimum turning radius $r_{m}$ for all feasible $\theta_{s}$ and $\theta_{f}$ is given as:

$d_{\text {min }}= \begin{cases}4 r_{m} \sqrt{1-2 \cos (\pi-\psi / 2)}, & \text { if case } 1 \text { or } 2 \\ 2 r_{m}, & \text { otherwise. }\end{cases}$

Thus, two configurations whose Euclidean distance between is greater than $d_{\min }$ satisfy one of conditions for having a solution. However, note that this is a necessary condition since there are other boundary conditions to be satisfied as defined through this section. Besides, configurations which make linear paths need to be handled as exceptions as they require infinite turning radius or zero curvature.

The proposed path shaping algorithm satisfies the kinematic constraint of the UAV with only one discontinuity at the intermediate vertex, and can shape the path (or equivalently, change the path length) using one parameter $\phi_{s}$ with its boundary condition according to the initial and final configurations. This technique will be applied to coordinated standoff target tracking which makes two UAVs arrive at a desired orbit and angular separation from the following section.

\section{Coordinated Standoff TARget TRACKIng Using PATH SHAPING APPROACH}

This section describes the coordinated standoff tracking for a stationary target and a moving target whose maneuver can be predicted precisely using the proposed path shaping technique. A conventional simultaneous arrival (SA) concept for two UAVs to reach the desired standoff orbit at the same time is first introduced. And then, a two-orbit approach is newly proposed in which UAVs first arrive at the outer orbit and subsequently shrink to the desired inner orbit at different time, adjusting the angular separation between UAVs, in order to address arrival time delay or failure of the UAV.

\section{A. Simultaneous arrival}

Given initial positions and orientations for UAVs and target information, it is possible to determine two arrival points satisfying the standoff distance as well as the desired angular separation between UAVs in terms of the line of sight angle 0 relative to the target. However, such a goal can be satisfied fully only if the two vehicles reach the respective position at the same time, while the path shaping method does not provide any control on the arrival time directly. Assuming the constant velocity of the vehicle, the time required to get to the designated position proportional to the path length with the speed. Thus, if there are no errors and disturbances in the system, the arrival time can be determined explicitly by adjusting the curvature of the path. In path shaping using constant curvature segments, the arc length is simply obtained by using the curvature $\kappa$ and the turning angle $\theta$ as:

$$
l_{a}=\theta / \kappa .
$$

By using the formulas given in Sec. II, the path length $l_{s f}$ between initial and final positions can be expressed as:

$$
l_{s f}=\frac{d}{2}\left[\frac{\sin \left(\phi_{f}-\gamma_{f}\right)}{\sin \phi_{s}} \theta_{s}+\frac{\sin \left(\phi_{s}-\gamma_{s}\right)}{\sin \phi_{f}} \theta_{f}\right]
$$


where $\phi_{s}$ is the only independent variable, which can be used as a tuning parameter for the path length. Noting that $\left(\phi_{f}, \theta_{s}, \theta_{f}\right)$ vary almost linearly with $\phi_{s}$, and its valid range is usually not too wide because of the physical constraints, the path length function $l_{s f}$ has a nearly-linear behavior. Defining squared path length difference as a performance index, optimal feasible solution $\phi_{s}$ for two UAVs can be obtained solving a constrained nonlinear minimization problem, given as:

$$
\left(\phi_{s_{1}}^{*}, \phi_{s_{2}}^{*}\right)=\arg \min _{\left(\phi_{s_{1}}, \phi_{s_{2}}\right)}\left(l_{s f_{1}}-l_{s f_{2}}\right)^{2}
$$

where subscript represents value for the $i$-th UAV. In case that path length ranges for the two vehicles partially overlap, there are many solutions providing the same path length (i.e. same arrival time). Depending on the task objectives, either the shortest path solution or the one requiring the smallest curvature variation can be chosen.

\section{B. Phase correction by a two-orbit approach}

When initial positions of two UAVs are far away, the simultaneous arrival is not possible to achieve, or demands too much energy due to keep the time constraint resulting in the long detour of one of the UAVs. Moreover, to minimze the exposure time of UAVs in the enemy radar site while maximizing the effect of simultaneous arrival to the designated position, the mission planner should be able to cope with the failure of the UAV or the arrival time delay by wind or unexpected obstacles during the mission as illustrated in Fig. 8(a). To address aforementioned problems, a two-orbit approach for the phase control is introduced as shown in Fig. 8(b). The idea is to reach the observation (or outer) orbit first, and then to perform a further (referred in the following as 'shrink') maneuver identical for two UAVs that brings the UAVs on an inner orbit at different time. The difference of time spent on the outer orbit allows for the control of the angular separation of the UAVs.

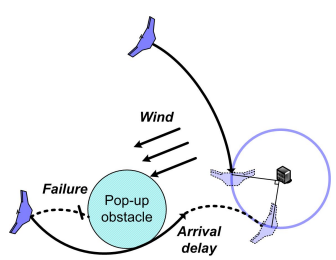

(a) Simultaneous arrival

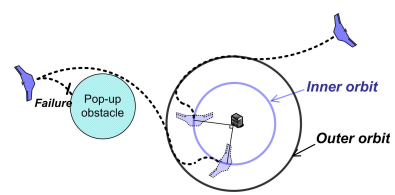

(b) Two-orbit approach
Fig. 8. Motivation of a two-orbit approach

Once one of UAVs reaches the outer orbit, that UAV remains on the same orbit until the other UAV reaches the orbit. Let us define the time $t_{r}$ as the time both UAVs get on the outer orbit and leader and follower based on the relative phase angle with respect to the target as given:

$$
\text { Leader }=\arg \max _{U A V_{i}}\left(\sigma_{U A V_{1}}\left(t_{r}\right), \sigma_{U A V_{2}}\left(t_{r}\right)\right)
$$

where $\sigma_{U A V_{i}} \in[0,2 \pi)$ represents the phase angle of the $i$-th UAV. The shrink maneuver is illustrated as shown in Fig. 9. Given the desired standoff distance which is equivalent to the inner orbit radius $r_{i}$, parameters to be chosen are an outer

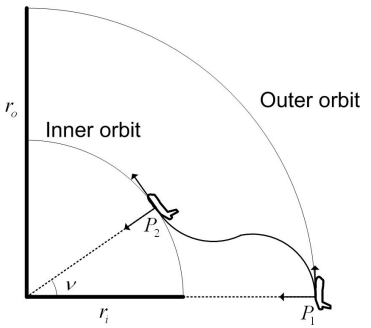

Fig. 9. An illustration of the two-orbit approach

orbit radius $r_{o}$ and an approach angle $\nu$. Then, the arrival point $P_{2}$ on the inner orbit and the orientation angle are uniquely defined given these values, and the path shaping technique using constant curvature segments is applied to connect configuration $P_{1}$ and $P_{2}$. It is worth noting that since the shrink maneuver itself represents a sort of an additional path, $\nu$ should be designed to make the path as short as possible. Moreover, the smaller value of $r_{i}$ closer to the outer orbit radius will lead to a smaller time needed for the shrink maneuver, however, on the other hand, it will take more time to achieve the desired angular separation between UAVs; thus, parameter $r_{o}$ and $\nu$ should be determined considering a tradeoff between these two aspects. To achieve the desired angular separation, let us define the phase difference between UAVs at time $t_{r}$ as:

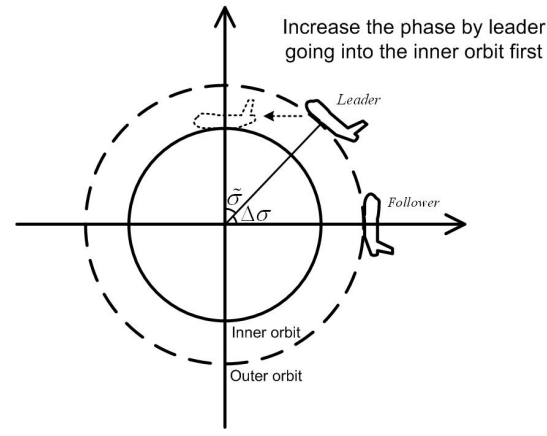

(a) Leader entering the inner orbit

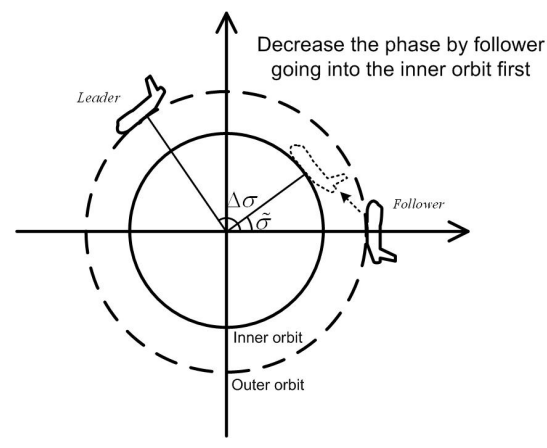

(b) Follower entering the inner orbit

Fig. 10. Phase correction by one of UAVs depending on the situation

$$
\begin{aligned}
\triangle \sigma & =\sigma_{l}-\sigma_{f}, & & \text { if } \sigma_{l}-\sigma_{f} \leq \pi \\
& =\sigma_{l}-\sigma_{f}-2 \pi, & & \text { otherwise }
\end{aligned}
$$

where $\sigma_{l}$ and $\sigma_{f}$ represent the phase angle of leader and follower at time $t_{r}$ with respect to the target, respectively. 
Then, the phase angle error $\tilde{\sigma}$ which needs to be corrected by the two-orbit approach is defined as:

$$
\tilde{\sigma}=\left(\sigma_{d}-|\triangle \sigma|\right) \operatorname{sign}(\triangle \sigma)
$$

where $\sigma_{d}$ is the desired angular separation. If the phase angle error $\tilde{\sigma}$ is positive, the leader should enter the inner orbit first to increase the angular separation from $\triangle \sigma$ to the $\sigma_{d}$. Similarly, if $\tilde{\sigma}$ is negative, the follower will shrink to the inner orbit first to decrease the angular separation. Figure 10 shows an exemplary decision as to the UAV entering the inner orbit first in case of $\sigma_{d}=\pi / 2$. Once one appropriate UAV reaches the inner orbit by the shrink maneuver, the other UAV needs to wait for a certain amount of time before starting the shrink maneuver. Considering the UAV flying on the orbit of radius $r$ with a constant speed $\mathrm{V}$, the angular variation $\alpha$ during time $t$ is given:

$$
\alpha=V t / r .
$$

Thus, time $T_{c}$ needed to correct the phase difference $\triangle \sigma$ to the desired value by the two different orbit can be obtained as:

$$
\begin{aligned}
|\tilde{\sigma}| & =V T_{c} / r_{i}-V T_{c} / r_{o} \\
T_{c} & =\left\{|\tilde{\sigma}| r_{o} r_{i}\right\} /\left\{V\left(r_{o}-r_{i}\right)\right\} .
\end{aligned}
$$

The aforementioned path shaping algorithm is for the stationary target in general. However, it can be extended to the moving target case in a certain situation such that the update rate of the target information is very low, expected future information for a lost target is given, or target movement can be predicted precisely. In those situations, the objective of the path shaping could be the gathering of UAVs to the appropriate position on the standoff orbit with the desired angular separation considering target movement. For this, let us first define the maximum time for the phase angle correction $T_{c_{\max }}$ with the relation $|\tilde{\sigma}| \leq \sigma_{d}$ from Eq. (58) as:

$$
T_{c_{\max }}=\frac{|\tilde{\sigma}|_{\max } r_{o} r_{i}}{V\left(r_{o}-r_{i}\right)}=\frac{\sigma_{d} r_{o} r_{i}}{V\left(r_{o}-r_{i}\right)} .
$$

When both UAVs reach the outer orbit of the expected future target position at time $t_{d}$, the remaining time $t_{\text {rem }}$ should be greater than the time needed for the shrink maneuver of both UAVs and the phase angle correction $T_{c_{\max }}$ as given:

$$
t_{\text {rem }}=t_{d}-t_{a} \geq 2 t_{s h}+T_{c_{\max }}
$$

where $t_{a}$ represents the latest arrival flight time of UAVs to the outer orbit from the initial positions, and $t_{s h}$ represents the time needed for shrink maneuver which can be obtained in advance by predetermined values of $r_{o}, r_{i}$ and $\nu$. Then, minimum time $t_{d}$ to gather UAVs around the expected target position at that time while keeping the desired standoff distance and the angular separation is computed as:

$$
\begin{aligned}
\min t_{d} & \\
\text { s.t } t_{d} & \geq t_{a}+2 t_{s h}+T_{c_{\max }}
\end{aligned}
$$

Once cooperative UAVs achieve the goal (the standoff distance and the angular separation) using the path shaping approach, an active guidance algorithm such as the vector field guidance
[8], [10] can be applied to track the maneuvering target tightly while estimating information of the target through filtering as in [15], [34]. The target encirclement problem for a moving target and a workable solution that achieves uniform (temporal) separation of constant-speed vehicles is also considered in [35].

\section{NUMERICAL SIMULATIONS}

To verify the feasibility and benefits of the proposed approach in this paper, firstly, the path shaping using constant curvature segments is simulated using initial configuration $\left(\mathrm{x}, \mathrm{y}, \sigma_{s}\right)=(0,0,0)$ and final configuration $=(3,5$, $\left.\sigma_{f}=( \pm \pi / 4, \pm \pi / 2)\right)$ with bounds on curvature of $\kappa_{s}=1$ and $\kappa_{f}=1$. Figure 11 shows the examples of the paths using the solution $\phi_{s}$ minimizing the path length. This figure also shows the locus of all feasible intermediate vertex $\mathbf{v}_{i}$ with the corresponding possible paths. In Fig. 11(a), as $\phi_{s}$ increases, initial turning radius decreases and final turning radius increases confirming Lemma II.1 and II.2 for the first case of path solutions. Similarly, as $\phi_{s}$ increases, both initial and final turning radius decreases for the third and fourth case as shown in Fig. 11(c) $\sim(d)$.

Figure 12 compares the average path length and curvature difference at intermediate vertex (or discontinuous points when the segment changes from arc to straight line for the Dubins path) between the proposed path shaping and Dubins path (see [31] for the path generation). Simulations are performed a hundred times for each configuration set by changing final configuration $\left(E \cos \theta_{m}, E \sin \theta_{m}, \sigma_{f, \text { rand }}\right)$ for all $5 \leq E \leq 15$ and $0 \leq \theta_{m}<2 \pi$ with fixed initial configuration $(0,0,0)$ and a bound on curvature of one, where $\sigma_{f, \text { rand }}$ represents a randomly generated angle. As a Euclidean distance $E$ between two points increases, the average path length of the proposed method gets longer than that of the Dubins path, and curvature difference decreases whereas that of Dubins remains the same. Consequently, it can be conjectured that the proposed algorithm provides not only fewer discontinuous points (which is one for the proposed method and two for the Dubins path), but also less discontinuity (i.e. curvature difference) since as can be seen for case 3 and 4, curvature differences are much smaller than those of the Dubins paths at the expense of the path length.

Moreover, to apply the two-orbit approach, given the constant velocity of $40 \mathrm{~m} / \mathrm{s}$ and the desired inner orbit radius of $200 \mathrm{~m}$, the appropriate value for the shrinking maneuver is investigated with different approach angle $v$ and orbit radius ratio $\beta=r_{o} / r_{i}$ as shown in Fig. 13. As explained earlier, parameter $\beta$ and $v$ should be determined considering a tradeoff between two aspects, which are the additional path length due to the shrink maneuver and the time required for the phase correction. To get a reasonable phase correction time, the orbit radius ratio $\beta$ can be suggested to be greater than two as shown Fig. 13(d), and then $v$ can be determined accordingly as shown in Fig. 13(c). Figure 14 shows the path shaping result for the coordinated standoff tracking of the stationary target with $V=40 \mathrm{~m} / \mathrm{s}, r_{i}=200 \mathrm{~m}, \beta=2, v=20^{\circ}$ and $\sigma_{d}=\pi / 2$. At time $t_{r}$, both of UAVs reach the outer orbit, 


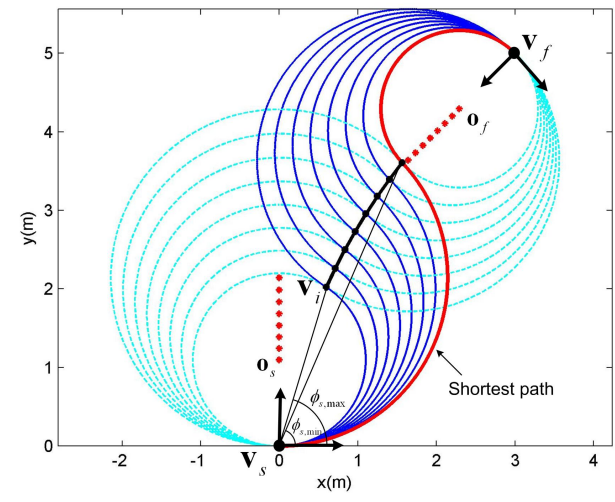

(a) First case

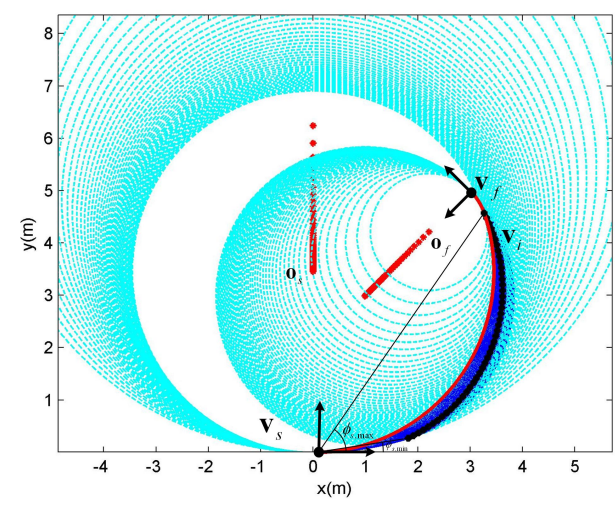

(c) Third case

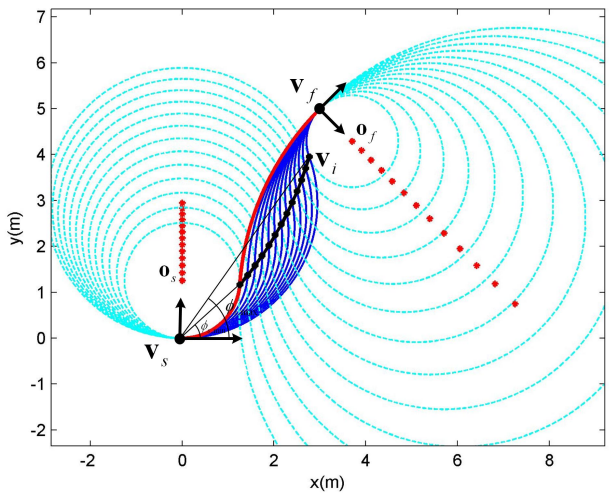

(b) Second case

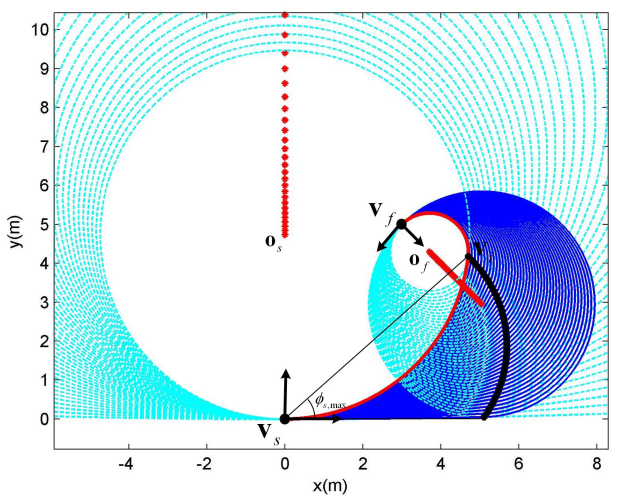

(d) Fourth case

Fig. 11. Examples of path using constant curvature segments $\left(\left(\kappa_{s}, \kappa_{f}\right)=(1,1)\right.$, blue lines are possible paths, and red dots represent the trace of the center of each curvature segment according to $\phi_{s}$.)

and then the follower $\left(\mathrm{UAV}_{1}\right.$ which reaches the outer orbit later) first enters into the inner orbit to decrease the phase angle difference between UAVs to a desired value $\sigma_{d}$. After $T_{c}$ seconds, the leader goes into the inner orbit using the same shrink maneuver as that of the follower. At 34 seconds, both the standoff distance $\left(r_{i}=200 \mathrm{~m}\right)$ from the target and desired angular separation $\left(\sigma_{d}=\pi / 2\right)$ between two UAVs are satisfied at the same time as shown in Fig. 14. The path shaping result for the coordinated standoff tracking of the moving target is shown in Fig. 15. The speed of UAV is set to $40 \mathrm{~m} / \mathrm{s}$ and target $10 \mathrm{~m} / \mathrm{s}$. Minimum time $t_{d}$ to gather UAVs around the target is computed firstly, and the similar approach as the stationary target case is applied with the expected position of target at that time. At 53 seconds, both UAVs are gathered while satisfying the desired standoff distance from the target and angular separation between them.

\section{Conclusions}

This paper has presented a novel path shaping approach for coordinated standoff target tracking using multiple UAVs. To reflect the fact that fixed-wing UAVs fly efficiently at a nominal airspeed, the path shaping approach was proposed for standoff tracking, which initialises UAVs on a standoff orbit with a desired angular separation while holding a constant velocity. For this, this paper introduced the use of two constant curvature segments having more flexibility and fewer discontinuous points on a curvature command compared to the Dubins path at the expense of the path length as well as satisfying the turning constraint of the UAV. Moreover, a simultaneous arrival concept was first introduced, and then a phase correction with shrink maneuver was newly proposed to address arrival time delay or failure of the UAV. To apply the two-orbit approach, given the constant velocity and the desired inner orbit radius, the appropriate value of the shrinking maneuver was investigated with different approach angle and orbit radius ratio. Numerical simulations for both stationary and moving ground targets using two cooperative UAVs showed the feasibility and properties of the proposed the two-orbit algorithm achieving the desired goal. As future work, the proposed standoff tracking strategy will be extended by considering more than two UAVs and a partially known moving ground target by predicting its future trajectory.

\section{REFERENCES}

[1] H. Choi, Y. Kim, and H. Kim. Genetic algorithm based decentralized task assignment for multiple UAVs in dynamic environments. International Journal of Aeronautical and Space Sciences, 12(2):163-174, 2011.

[2] J.D. Coker and A.H. Tewfik. Performance synthesis of UAV trajectories in multistatic SAR. IEEE Transactions on Aerospace and Electronic Systems, 47(2):848-863, 2011. 


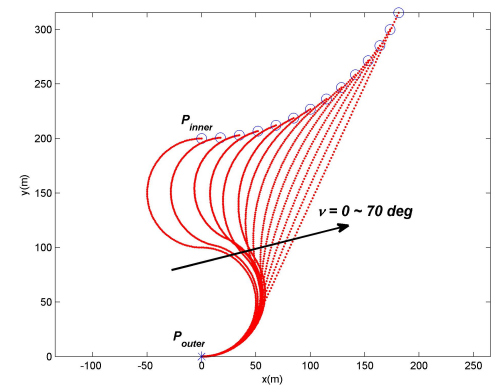

(a) Example trajectory of shrink maneuver $\left(\beta=\frac{r_{o}}{r_{i}}=2\right)$

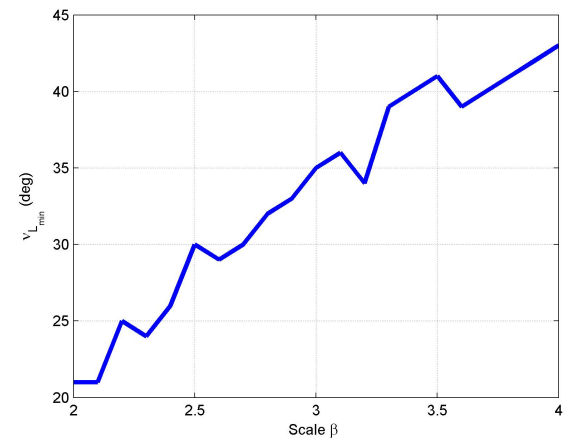

(c) Approach angle $\nu$ having minimum path length

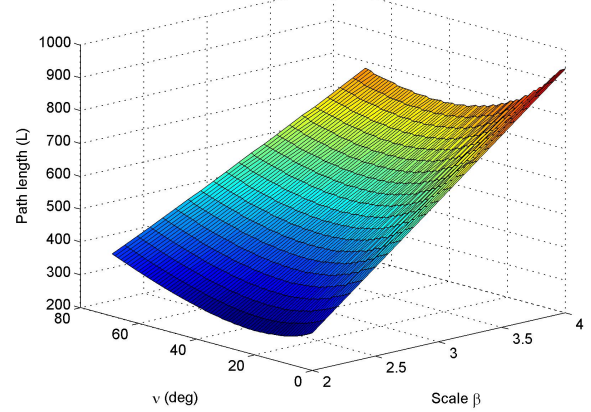

(b) Path length

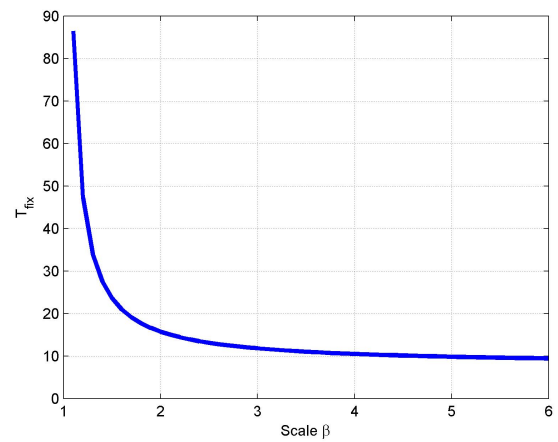

(d) Time for the phase correction $(\tilde{\sigma}=\pi / 2)$

Fig. 13. Effect on shrink maneuver of different parameters $\left(V=40 \mathrm{~m} / \mathrm{s}, r_{i}=200 \mathrm{~m}\right)$

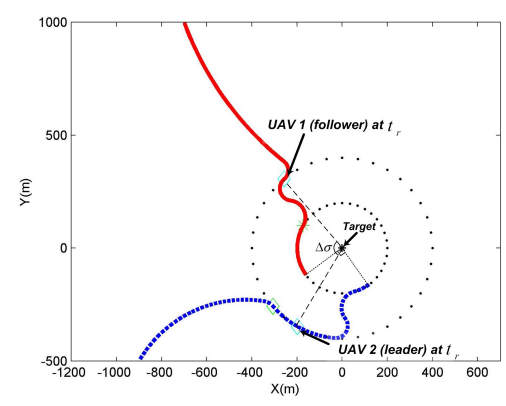

(a) Trajectory of two UAVs

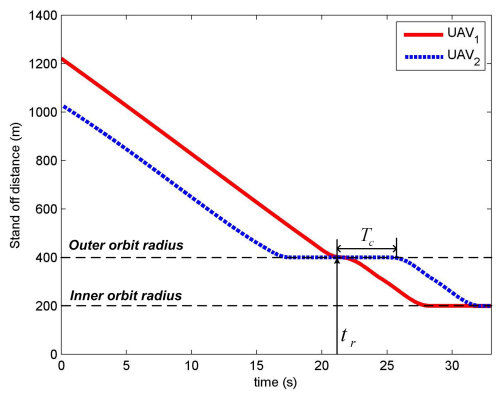

(b) Standoff distance from the target

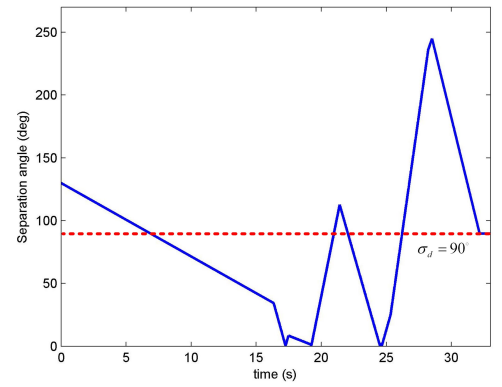

(c) Angular separation between two UAVs

Fig. 14. Path shaping result for coordinated standoff tracking of the stationary target

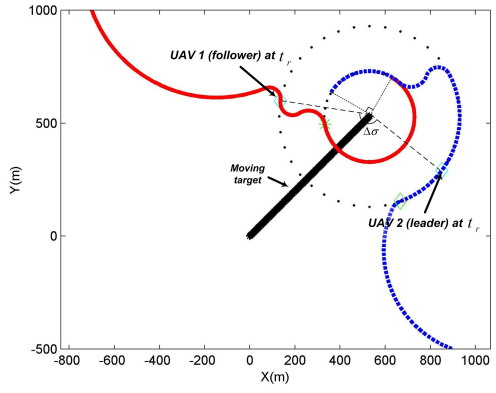

(a) Trajectory of two UAVs

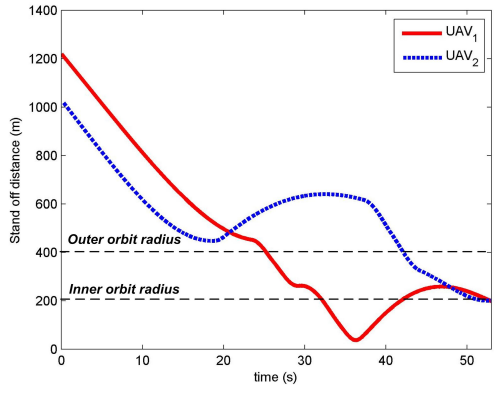

(b) Standoff distance from the target

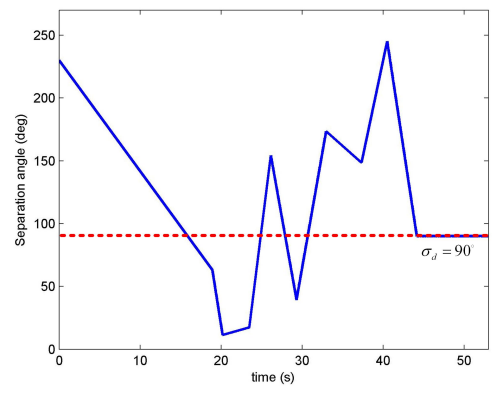

(c) Angular separation between two UAVs

Fig. 15. Path shaping result for coordinated standoff tracking of the moving target 


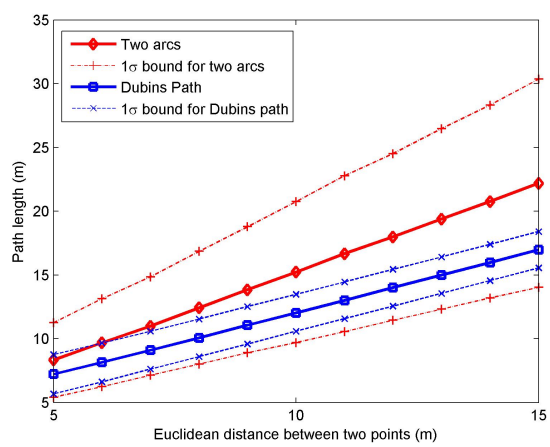

(a) Path length

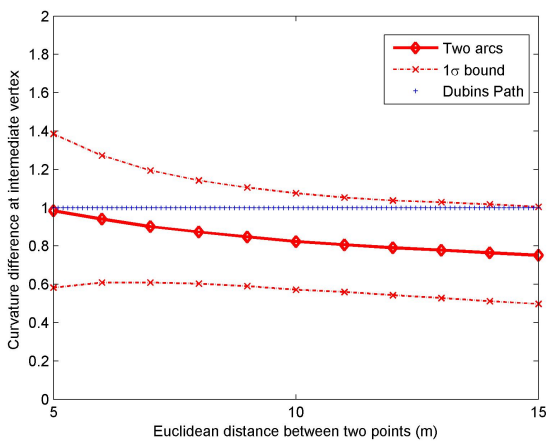

(b) Curvature difference

Fig. 12. Comparison between the proposed method and Dubins path

[3] J.R. Riehl, G.E. Collins, and J.P. Hespanha. Cooperative search by UAV teams: A model predictive approach using dynamic graphs. IEEE Transactions on Aerospace and Electronic Systems, 47(4):2637-2656, 2011.

[4] Z. Pengcheng, D.W. Casbeer, and A.L. Swindlehurst. Adaptive mobile sensor positioning for multi-static target tracking. IEEE Transactions on Aerospace and Electronic Systems, 46(1):120-132, 2010.

[5] S. Martinez and F. Bullo. Optimal sensor placement and motion coordination for target tracking. Automatica, 42(4):661-668, 2006.

[6] G. Gu, P. Chandler, C. Schumacher, A. Sparks, and M. Pachter. Optimal cooperative sensing using a teams of UAVs. IEEE Transactions on Aerospace and Electronic Systems, 42(4):1446-1458, 2006.

[7] E.W. Frew. Sensitivity of cooperative target geolocalization to orbit coordination. Journal of Guidance, Control, and Dynamics, 31(4):10281040, 2008.

[8] E.W. Frew, D.A. Lawrence, and S. Morris. Coordinated standoff tracking of moving targets using Lyapunov guidance vector fields. Journal of Guidance, Control, and Dynamics, 31(2):290-306, 2008.

[9] T.H. Summers, M.R. Akella, and M.J. Mears. Coordinated standoff tracking of moving targets: Control laws and information architectures. Journal of Guidance, Control, and Dynamics, 32(1):56-69, 2009.

[10] D. Kingston and R. Beard. UAV spaly state configuration for moving targets in wind. Lecture Notes in Control and Information, 369:109-128, 2007.

[11] R. Sepulchre, D.A. Paley, and N.E. Leonard. Stabilization of planar collective motion: All-to-all communication. IEEE Transactions on Automatic Control, 52(5):811-824, 2007.

[12] D.A. Paley, N.E. Leonard, and R. Sepulchre. Stabilization of symmetric formations to motion around convex loops. Systems and Control Letters, 57:209-215, 2008.

[13] D.J. Klein and K.A. Morgansen. Controlled collective motion for trajectory tracking. In American Control Conference, American Control Conference, Minneapolis, Minnesota, 2006.

[14] R.A. Wise and R.T. Rysdyk. UAV coordination for autonomous target tracking. AIAA Guidance, Navigation and Control Conference, Keystone, Colorado, USA, 2006.

[15] S. Kim, H. Oh, and A. Tsourdos. Nonlinear model predictive coordinated standoff tracking of moving ground vehicle. Journal of Guidance, Control, and Dynamics, 36(2):557-566, 2013.
[16] H. Oh, S. Kim, A. Tsourdos, and B.A. White. Road-map assisted standoff tracking of moving ground vehicle using nonlinear model predictive control. In American Control Conference, Montreal, Canada, 2012.

[17] S.M. Malaek and A.R. Kosari. Novel minimum time trajectory planning in terrain following flights. IEEE Transactions on Aerospace and Electronic Systems, 43(1):2-12, 2007.

[18] L.E. Dubins. On curves of minimal length with a constraint on average curvature, and with prescribed initial and terminal positions and tangents. American Journal of Mathematics, 79(3):497-516, 1957.

[19] P. Chandler, S. Rasmussen, and M. Pachter. UAV cooperative path planning. In AIAA Guidance, Navigation, and Control Conference, Denver, CO, Aug. 1417 2000. AIAA-2000-4370.

[20] K. Yang S. Sukkarieh. 3D smooth path planning for a UAV in cluttered natural environments. In IEEE/RSJ International Conference on Intelligent Robots and Systems, Nice, France, Sep. 22-26 2008.

[21] U. Zengin and A. Dogan. Probabilistic trajectory planning for UAVs in dynamic environments. In AIAA 3rd Unmanned Unlimited Technical Conference, Workshop and Exhibit, Chicago, Illinois, Sep. 20-23 2004. AIAA 2004-6528.

[22] A. Richards and J.P. How. Aircraft trajectory planning with collision avoidance using mixed integer linear programming. In American Control Conference, Anchorage, AK, May. 8-10 2002.

[23] I.K. Nikolos, K.P. Valavanis, N.C. Tsourveloudis, and A.N. Kostaras Evolutionary algorithm based offline/online path planner for UAV navigation. IEEE Transactions on Systems, Man, and Cybernetics-Part B: Cybernetics, 33(6):898-912, 2003.

[24] E. Edison and T. Shima. Integrated task assignment and path optimization for cooperating uninhabited aerial vehicles using genetic algorithms. Computers \& Operations Research, 38(1):340-356, 2011.

[25] J.A. Reeds and R.A. Shepp. Optimal paths for a car that goes both forward and backward. Pacific Journal of Mathematics, 145(2):367393, 1990

[26] J. Boissonnat, A. Cérézo, and J. Leblond. Shortest paths of bounded curvature in the plane. Journal of Intelligent and Robotic System, 11:520, 1994.

[27] A.M. Shkel and V.J. Lumelsky. Classification of the Dubins set. Robotics and Autonomous Systems, 34:179-202, 2001.

[28] A. Tsourdos, B. White, and M. Shanmugavel. Cooperative Path Planning of Unmanned Aerial Vehicles. John Wiley \& Sons, 2010.

[29] S.M. LaValle. Planning Algorithms. Cambridge University Press, 2006.

[30] C. Tomlin, I. Mitchell, and R. Ghosh. Safety verification of conflict resolution manoeuvres. IEEE Transactions on Intelligent Transportation System, 2(2):110-120, 2001.

[31] M. Shanmugavel, A. Tsourdos, B. A. White, and R. Zbikowski. Differential geometric path planning of multiple UAVs. Journal of Dynamic Systems, Measurement, and Control, 129:620-632, 2007.

[32] M. Shanmugavel, A. Tsourdos, B. A. White, and R. Zbikowski. Cooperative path planning of multiple UAVs using dubins paths with clothoid arcs. Control Engineering Practice, 18(9):1084-1092, 2010.

[33] B.A. White, A. Tsourdos, I. Ashokaraj, S. Subchan, and R. Zbkowski. Contaminant cloud boundary monitoring using network of UAV sensors. IEEE Sensors Journal, 8(10):1681-1692, 2008.

[34] H. Oh, S. Kim, H.S. Shin, B.A. White, A. Tsourdos, and C.A. Rabbath. Rendezvous and standoff target tracking guidance using differential geometry. Journal of Intelligent and Robotic Systems, 69:389-405, 2013.

[35] Cameron Peterson and Derek A. Paley. Multivehicle coordination in an estimated time-varying flowfield. Journal of Guidance, Control, and Dynamics, 34(1):177-191, 2011. 

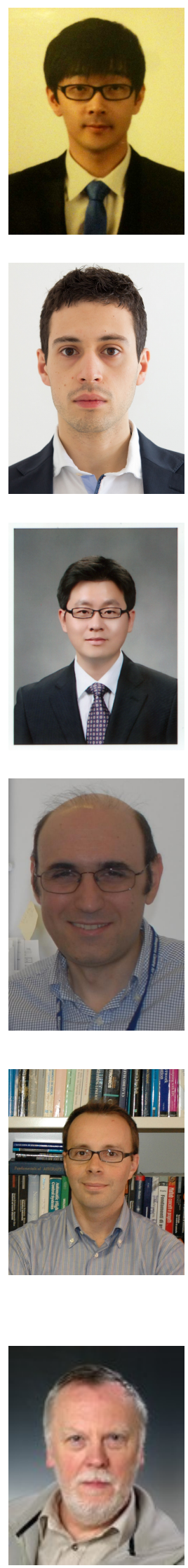

Hyondong Oh received the BSc. and MSc. degrees in Aerospace Engineering from KAIST, South Korea, in 2004 and 2010, respectively. And then he acquired a PhD on Autonomous Surveillance and Target Tracking Guidance Using Multiple UAVs from Cranfield University, UK in 2013. He is currently a postdoctoral research fellow at Nature Inspired Computing and Engineering (NICE) Group, Department of Computing, University of Surrey, UK. His research interests include biologically-inspired swarming robotics, cooperative control and path planning of autonomous systems, target surveillance and tracking guidance, and information/sensor fusion.

Dario Turchi received Laurea (BSc.) degree in Computer Engineering and Laurea Specialistica (MSc.) degree (cum Laude) in Control Engineering from University of Pisa, Pisa, Italy, in 2007 and 2011, respectively. Since 2013 he is a $\mathrm{PhD}$ student at the Department of Engineering Physics, School of Engineering, Cranfield University, UK. His research interests cover information consensus, sensor and data fusion, cooperative control and path planning of autonomous systems, guidance and navigation systems.

Seungkeun Kim received a BSc. degree in Mechanical and Aerospace Engineering from SNU(Seoul National University), Seoul, Korea, in 2002 and then acquired a PhD from SNU in 2008. He is currently an assistant professor at Dept of Aerospace Engineering, Chungnam National University, Korea. Previously he was a research fellow and a lecturer in Cranfield University, UK between Apr 2008 and Feb 2012. He has actively published book chapters, journal and conference papers related to unmanned systems. His research interests cover nonlinear guidance and control, estimation, sensor and information fusion, fault diagnosis, fault tolerant control, and decision making for unmanned systems.

Antonios Tsourdos obtained a MEng on Electronic, Control and Systems Engineering from the University of Sheffield (1995), an MSc on Systems Engineering from Cardiff University (1996) and a PhD on Nonlinear Robust Missile Autopilot Design and Analysis from Cranfield University (1999). He is a Professor of Control Engineering with Cranfield University. Appointed Head of the Autonomous Systems Group in 2007. Professor Tsourdos was member of the Team Stellar, the winning team for the UK MoD Grand Challenge (2008) and the IET Innovation Award (Category Team, 2009).

Lorenzo Pollini (IEEE Member since 1993) received the Laurea degree (cum laude) in computer engineering and the Ph.D. degree in Robotics and Industrial Automation from University of Pisa, Pisa, Italy, in 1997 and 2000, respectively. From 2000 to 2002, he was an Instructor of Automatic Control with the Italian Navy Academy. He is currently an Assistant Professor of automatic control with the School of Engineering, University of Pisa, Italy. His research interests concern guidance and navigation systems, vision based control, haptic support systems, fuzzy, nonlinear and adaptive control, real time dynamic systems simulation and embedded control systems development with specific application to unmanned systems.

Brian A. White received the B.Sc.Eng. degree from the University of Leicester, Leicester, U.K., in 1967 and the M.Sc. degree in automatic control and the Ph.D. degree in bond graph modeling from the University of Manchester Institute of Science and Technology, Manchester, U.K., in 1971 and 1973, respectively. He is a Professor Emeritus with Cranfield University, Cranfield, U.K. He is with the Autonomous Systems Group, Cranfield Defence and Security, Cranfield University. His research interests include robust control, nonlinear control, estimation, observer applications, inertial navigation, guidance design, soft computing, and sensor and data fusion. He was a member of Team Stellar, the winning team for the UK MoD Grand Challenge (2008) and the IET Innovation Award (Category Team, 2009) responsible for autonomous path planning of both the aerial and ground vehicles. 